\title{
Diabetic ketoacidosis and diabetes associated with antipsychotic exposure among a previously diabetes-naive population with schizophrenia: a nationwide nested case-control study
}

\author{
Christoffer Polcwiartek ${ }^{1,2,3,4}$ (iD $\cdot$ Kristian Kragholm ${ }^{1,2,3,4}$ - Christopher Rohde ${ }^{1,4}$. \\ Nasseh Hashemi $^{1,4}$ • Torkel Vang ${ }^{1,4}$ • Jimmi Nielsen ${ }^{1,4,5}$
}

Received: 20 February 2017 / Accepted: 9 May 2017 /Published online: 7 June 2017

(C) Springer-Verlag Berlin Heidelberg 2017

\begin{abstract}
Aims/hypothesis Diabetic ketoacidosis (DKA) is a potentially fatal metabolic emergency of both type 1 and type 2 diabetes. Although there is a reduced risk of type 1 diabetes in schizophrenia, the incidence of DKA is tenfold higher than that of the general population. Thus, we aimed to investigate associations between exposure to antipsychotic medication (within 3 months prior to event) and DKA, type 1 diabetes and type 2 diabetes. We also reported related, clinically relevant outcomes.

Methods Using a nested case-control study design, we identified cases of DKA, type 1 diabetes and type 2 diabetes in a previously diabetes-naive population with schizophrenia in Denmark from 1995 to 2014. Cases were matched (by age, sex and year of schizophrenia onset) 1:5 to schizophrenic control individuals who were alive and had not emigrated prior to event. Conditional logistic regression was used to compute ORs with $95 \%$ CIs. Other outcomes included diabetes aetiology of DKA, in-hospital mortality, DKA readmissions and temporal trends of use of insulin and oral glucoselowering agents.
\end{abstract}

Christoffer Polcwiartek

c.polcwiartek@gmail.com

1 Department of Psychiatry, Aalborg University Hospital, Brandevej 5, 9220 Aalborg, Denmark

2 Department of Cardiology, Aalborg University Hospital, Aalborg, Denmark

3 Department of Clinical Epidemiology and Biostatistics, Aalborg University Hospital, Aalborg, Denmark

4 Department of Clinical Medicine, Aalborg University, Aalborg, Denmark

5 Mental Health Centre Glostrup, Copenhagen University Hospital, Copenhagen, Denmark
Results Of 29,955 individuals with schizophrenia, we identified 28 individuals with DKA, 90 with type 1 diabetes and 2140 with type 2 diabetes. These were matched to 137 , 410 and 9861 individuals in the control group, respectively. Antipsychotic exposure was associated with DKA (OR 2.60; 95\% CI 1.06, 6.38) and type 2 diabetes (OR 1.64; $95 \%$ CI 1.48, 1.83). A trend towards increased risk of type 1 diabetes was found but remained insignificant (OR 1.38; $95 \%$ CI $0.84,2.29$ ). Diabetes aetiology of DKA was type 1 in eight cases and type 2 in 14 cases. Of the remaining six cases of DKA, aetiology could not be determined, as four were fatal within 8 days and for two, no prescriptions for insulin and oral glucose-lowering agents were redeemed. Of all DKA cases, six had more than one episode of DKA, and of all type 1 diabetes and type 2 diabetes cases, four and 11, respectively, had at least one episode. Use of insulin and oral glucose-lowering agents was higher among individuals with DKA relative to those with type 1 diabetes and type 2 diabetes.

Conclusions/interpretation Antipsychotic exposure was associated with DKA and type 2 diabetes in a previously diabetes-naive schizophrenia population. Antipsychoticassociated DKA is relevant not only for psychiatrists but also for other physicians who may manage and admit such patients.

Keywords Antipsychotics - Diabetic ketoacidosis · Internal medicine $\cdot$ Mortality $\cdot$ Schizophrenia $\cdot$ Type 1 diabetes $\cdot$ Type 2 diabetes
Abbreviations
ATC
Anatomical Therapeutic Chemical
CCI
Charlson Comorbidity Index
DDD Defined Daily Dose 
DKA Diabetic ketoacidosis

FGA First-generation antipsychotic

LADA Latent autoimmune diabetes in adults

p25-p75 25th and 75th percentiles

SGA Second-generation antipsychotic

\section{Introduction}

Schizophrenia is associated with a more than 20 year reduction in expected lifespan relative to that of the general population; reasons for this excess mortality are multifactorial [1]. Important factors include the broad occurrence of unhealthy lifestyle habits, undertreatment of physical conditions, lack of regular health examinations and the possibility that exposure to antipsychotic medication may pose a greater risk of cardiometabolic complications, including type 2 diabetes [2-4]. Diabetic ketoacidosis (DKA), which is a metabolic emergency of both type 1 and type 2 diabetes, may progress to coma and death within a few hours if not managed properly. Poor adherence to insulin and oral glucose-lowering agents and acute medical conditions are considered the major precipitating factors for DKA [5]. However, exposure to antipsychotics, especially second-generation antipsychotics (SGAs), has also been associated with the condition [6]. Notably, the incidence of DKA in the population with schizophrenia exposed to antipsychotics is tenfold higher relative to the general population [7]. Conversely, although DKA occurs predominantly in connection with type 1 diabetes, there is a reduced risk of type 1 diabetes among individuals with schizophrenia [8-11].

Antipsychotic-associated DKA is clinically relevant not only for psychiatrists but also for general practitioners, emergency physicians, internal medicine physicians such as endocrinologists, and other physicians who initially may manage the DKA emergency and/or facilitate admission to a hospital ward. Occasionally, DKA may be the initial presentation of new-onset diabetes in the schizophrenia population, with mortality as high as $26.5 \%$ [11]. In addition, $\mathrm{HbA}_{1 \mathrm{c}}$ levels are often increased for at least several weeks prior to the onset of DKA, further underscoring challenges with underdiagnosed diabetes in this susceptible population [7]. Of further concern, young individuals with type 1 diabetes exposed to antipsychotics are found to have poor glycaemic control and increased risks of severe hypoglycaemia and DKA [12]. Clearly, a better understanding of the development of antipsychotic-associated DKA is warranted to potentially improve prevention, early recognition, treatment and, consequently, prognosis.

For these reasons, we aimed to investigate associations between antipsychotic exposure (within 3 months prior to event) and DKA, type 1 diabetes and type 2 diabetes among a previously diabetes-naive population with schizophrenia. We further reported diabetes aetiology of DKA, in-hospital mortality and long-term outcomes such as DKA readmissions and temporal trends of use of insulin and oral glucoselowering agents.

\section{Methods}

Study design and registers We performed a nationwide register-based nested case-control study from 1 January 1995 to 31 December 2014. This study period was chosen to allow full data retrieval from all used registers. Statistics Denmark and the Danish Health Data Authority approved use of the data for the study. Ethics approval is not required for retrospective register-based studies in Denmark.

Data were obtained by confidentially cross-linking nationwide healthcare registers using the unique personal civil registration number, ensuring accurate linkage of data between registers. The Danish Civil Registration System from 1968 was used to obtain data on birth date, sex and migrations [13]. The Danish Psychiatric Central Research Register from 1969 was used to obtain data on psychiatric admissions and diagnoses, where ICD-8 was used until 1994 and thereafter replaced by ICD-10 (www. who.int/classifications/icd/en/) [14]. The Danish National Patient Register from 1977 was used to obtain data on admissions and diagnoses for physical conditions, where ICD-8 and ICD-10 also were used [15]. The Danish National Prescription Register from 1995 was used to obtain data on prescription-based medications, where type of medication was registered according to Anatomical Therapeutic Chemical (ATC) codes, and dose of medication was given as a Defined Daily Dose (DDD) [16]. The Danish Register of Causes of Death from 1970 was used to obtain data on mortality [17].

Cases and controls Only those diagnosed with schizophrenia (ICD-10: F20.x) in the Danish Psychiatric Central Research Register were included. This population was chosen as it is at increased risk of DKA [7] and indication of antipsychotic treatment is well established [18] relative to other populations, in which development of diabetes and potentially DKA may involve different mechanisms. Diagnoses from emergency room visits were disregarded because of low validity. Data on subtype, age at onset and duration of schizophrenia were reported, the latter being calculated as the time from onset of schizophrenia to mortality, emigration or end of the study on 31 December 2014, whichever came first.

Cases were defined as having an incident onset of DKA, type 1 diabetes or type 2 diabetes. DKA was defined according to one of the following ICD-10 discharge diagnoses: E1x.1, 
E10.0B, E11.0E or E12.0C. Type 1 diabetes was defined where at least one prescription for insulin (ATC: A10A) was redeemed, with use of ongoing monotherapy. Type 2 diabetes was defined where at least one prescription for an oral glucose-lowering agent (ATC: A10B) was redeemed, with use of ongoing monotherapy. This approach was applied as ICD-10 does not uniquely identify aetiology of diabetes, and some cases might have only been diagnosed by their general practitioner and, consequently, do not appear in the Danish National Patient Register. In Denmark, insulin monotherapy is only used as a treatment for type 2 diabetes in less than $5 \%$ of patients, so this definition was considered valid [19].

Controls were selected using a risk-set sampling strategy. Each individual was matched by age, sex and year of schizophrenia onset to up to five randomly selected schizophrenic control individuals who were alive and had not emigrated prior to event. Controls were assigned an index date identical to that of the corresponding case.

The index date was defined as the time of incident onset of DKA, type 1 diabetes or type 2 diabetes in the Danish National Patient Register, or the time of redeeming a first prescription for insulin or an oral glucose-lowering agent in the Danish National Prescription Register, whichever came first.

Cases and controls with a prior history of pancreatitis (ICD-8: 577; ICD-10: K85-86), pancreatic cancer (ICD-8: 157; ICD-10: C25) or polycystic ovary syndrome (ICD-8: 256.9; ICD-10: E28.2) were excluded, as these conditions may involve different mechanisms for developing diabetes and potentially DKA.

Exposure definition Antipsychotic exposure (within 3 months prior to event) was defined as redeeming at least one prescription for an antipsychotic medication (ATC: N05A except lithium N05AN01) in the Danish National Prescription Register. Antipsychotics were categorised as first-generation antipsychotics (FGAs) and SGAs, as previously described [20]. Data on antipsychotic polypharmacy (i.e. exposure to more than one antipsychotic) were reported, and antipsychotic dose was calculated as the amount of DDD. As medication status during inpatient admission was not available as part of the Danish National Prescription Register, the number of psychiatric bed days was excluded from the calculation.

Outcome measures The primary outcomes were DKA, type 1 diabetes and type 2 diabetes. Secondary outcomes were diabetes aetiology of DKA (i.e. post-DKA diagnosis of type 1 diabetes or type 2 diabetes), in-hospital mortality and longterm outcomes such as DKA readmissions and temporal trends of use of insulin and oral glucose-lowering agents. The latter was examined by calculating the total number of annual redeemed prescriptions for insulin and oral glucoselowering agents and the lifetime cumulative amount of DDDs.
Statistical analysis Categorical variables were reported as frequencies with corresponding percentages, and continuous variables as medians with corresponding 25 th and 75 th percentiles (p25-p75). Differences between cases of post-DKA type 1 diabetes vs type 1 diabetes and cases of post-DKA type 2 diabetes vs type 2 diabetes were examined. Analyses were carried out using the non-parametric Mann-Whitney $U$ test for continuous variables, and Fisher's exact or the $\chi^{2}$ test for categorical variables.

Conditional logistic regression was used to examine associations between antipsychotic exposure and DKA, type 1 diabetes and type 2 diabetes. Crude and adjusted ORs with corresponding 95\% CIs were computed, with no antipsychotic exposure as reference. Adjustments were made for the following potential confounders known or suspected to modify any diabetic condition: (1) Charlson Comorbidity Index (CCI), where we disregarded diagnoses included in the CCI established less than 1 year prior to event and defined the level of physical comorbidity as none (CCI score 0 ), low (CCI score 1) and moderate/high (CCI score $\geq 2$ ) [21]; (2) diabetogenic co-medication exposure (within 3 months prior to event), including thiazides (ATC: C03A), beta blockers (ATC: C07), statins (ATC: C10AA), oral contraceptives (ATC: G03A), glucocorticoids (ATC: H02AB) and calcineurin inhibitors (ATC: L04AD); (3) psychotropic co-medication exposure (within 3 months prior to event), including antiepileptics (ATC: N03A except clonazepam N03AE01), anticholinergics (ATC: N04A), lithium (ATC: N05AN01), benzodiazepines (ATC: N05BA, N05CD, N05CF, N03AE01) and antidepressants (ATC: N06A). As age and sex were handled by the matching procedure, these potential confounders were not included in adjusted analyses. Co-medication exposure was used as a categorical variable only including integer numbers (e.g. two co-medications yielded twice the listed OR estimate).

Data management and analysis were performed using Stata software, version 14.1 (StataCorp, College Station, Texas, USA), and R software, version 3.3.2 (R Development Core Team, Vienna, Austria). All analyses were two-sided; $p$ values $<0.05$ were considered statistically significant.

\section{Results}

Characteristics of cases and controls Of 29,955 individuals from the previously diabetes-naive schizophrenia population, we identified 28 cases of incident DKA, 90 of type 1 diabetes and 2140 of type 2 diabetes during the study period. These were matched to 137,410 and 9861 controls, respectively. Fig. 1 depicts a flowchart showing the inclusion and exclusion of cases and controls, and Tables 1, 2 and 3 show the characteristics of the cases and controls.

Most cases were adults over 18 years, with the exception of six type 2 diabetes cases $(0.3 \%)$. DKA cases were characterised 


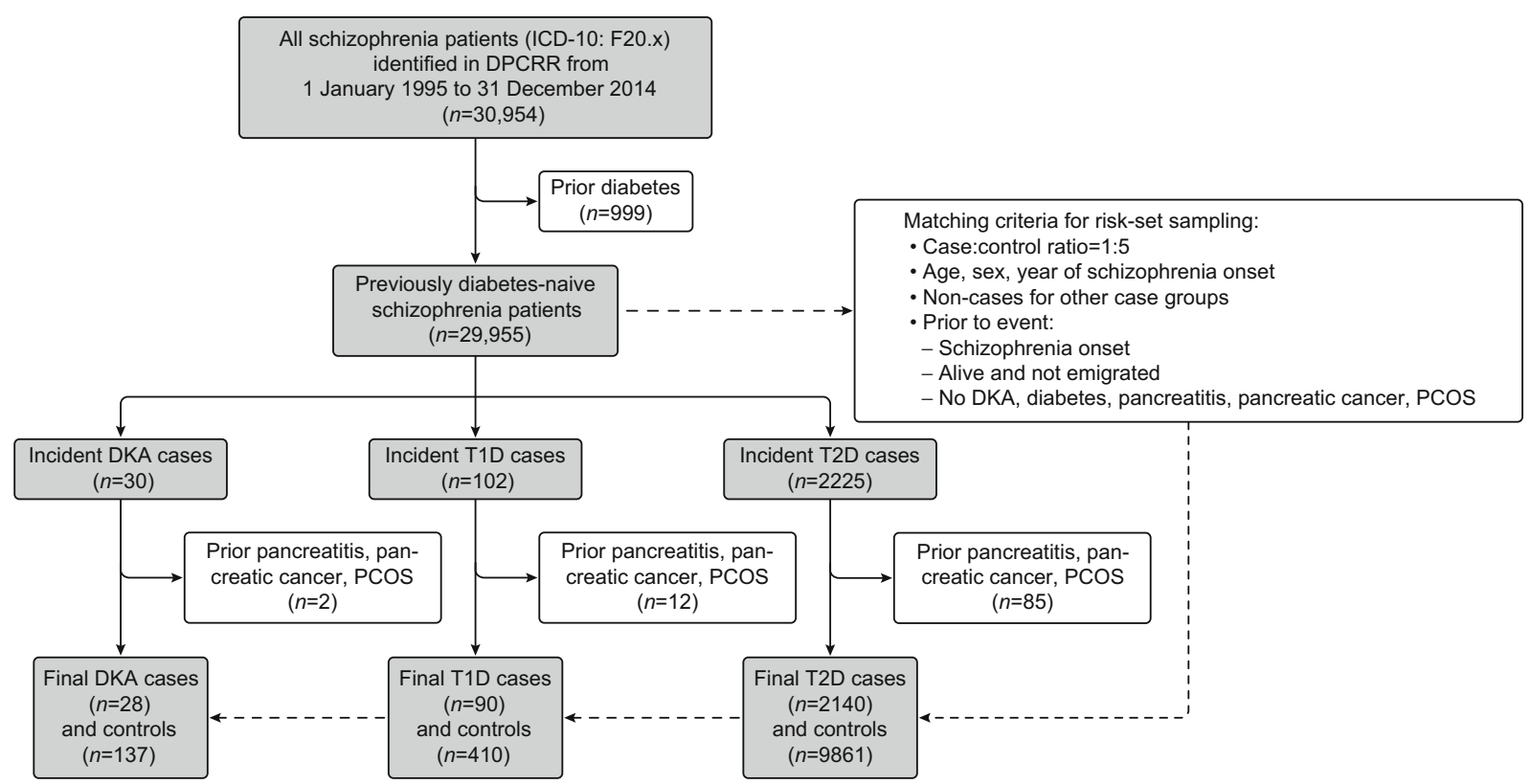

Fig. 1 Flowchart depicts the process of inclusion and exclusion of cases and controls using risk-set sampling. DPCRR, Danish Psychiatric Central Research Register; PCOS, polycystic ovary syndrome; T1D, type 1 diabetes; T2D, type 2 diabetes

by a higher proportion of males, lower median age and longer duration of schizophrenia relative to cases of type 1 diabetes and type 2 diabetes. Relative to controls, cases were more often exposed to antipsychotics and antipsychotic polypharmacy and received a higher DDD of antipsychotics. On a group level, cases were often associated with exposure to low-potency FGAs, clozapine, olanzapine, quetiapine and risperidone. In addition, the included potential confounders such as increased CCI score and exposure to several diabetogenic and psychotropic co-medications were more frequent among cases.

Risks of DKA and diabetes Antipsychotic exposure was associated with DKA (OR 2.60; 95\% CI 1.06, 6.38), as was increasing number of exposures to psychotropic co-medications (OR 4.06; 95\% CI 1.48, 11.11). Antipsychotic exposure tended towards an increased risk of type 1 diabetes but remained insignificant (OR 1.38; 95\% CI 0.84, 2.29). Conversely, antipsychotic exposure was associated with type 2 diabetes (OR 1.64; 95\% CI $1.48,1.83$ ); corresponding adjusted ORs yielded slightly lower estimates. Fig. 2 depicts the results of the conditional logistic regression.

Diabetes aetiology and in-hospital mortality of the 28 DKA cases, eight $(28.6 \%)$ were identified with post-DKA type 1 diabetes, of which five (17.9\%) were exposed to antipsychotics, and 14 (50.0\%) were identified with post-DKA type 2 diabetes, of which 10 (35.7\%) were exposed to antipsychotics. For these cases, median time to diabetes was 11 days (p25-p75: 8-13 days). Of the remaining six DKA cases $(21.4 \%)$, aetiology could not be determined, as four cases $(14.3 \%)$ were fatal within 8 days and, in two cases
(7.1\%), no prescriptions for insulin and oral glucoselowering agents were redeemed following resolution of DKA. Conversely, no in-hospital mortality among type 1 diabetes and type 2 diabetes cases was identified.

No significant differences were found between post-DKA type 1 diabetes cases $(n=8 / 28)$ vs type 1 diabetes cases $(n=90)$. Individuals with post-DKA type 1 diabetes tended to be younger at onset of schizophrenia $(p=0.058)$ and type 1 diabetes $(p=0.062)$ and to receive a lower DDD of antipsychotics $(p=0.055)$. Similarly, when comparing the post-DKA type 2 diabetes group ( $n=14 / 28$ ) vs the type 2 diabetes group $(n=2140)$, the former was significantly younger at onset of schizophrenia $(p=0.012)$ and type 2 diabetes $(p=0.002)$, received a lower DDD of antipsychotics $(p=0.012)$ and was more often exposed to oral contraceptives $(p=0.010)$. Table 4 shows the differences between cases of post-DKA type 1 diabetes vs type 1 diabetes and cases of post-DKA type 2 diabetes vs type 2 diabetes.

Long-term outcomes Of the 28 DKA cases, three (10.7\%) had more than one episode of DKA, two $(7.1 \%)$ had more than two episodes and one (3.6\%) had 14 episodes. Of the 90 individuals with type 1 diabetes, four (4.4\%) had an episode of DKA, of whom one (1.1\%) had more than one episode and one $(1.1 \%)$ had more than two episodes. Similarly, of the 2140 cases of type 2 diabetes, $11(0.5 \%)$ had an episode of DKA, of whom two $(0.1 \%)$ had more than one episode.

Annual redeemed prescriptions for insulin and oral glucoselowering agents increased linearly following event, as depicted in Fig. 3. All of the eight individuals with post-DKA type 1 diabetes received insulin monotherapy, with a median 
Table 1 Characteristics of incident DKA cases and their matched controls with schizophrenia

\begin{tabular}{|c|c|c|}
\hline Characteristic & DKA cases $(n=28)$ & Controls $(n=137)$ \\
\hline \multicolumn{3}{|l|}{ Demographics } \\
\hline Male sex & $21(75.0)$ & $102(74.5)$ \\
\hline Female sex & $7(25.0)$ & $35(25.5)$ \\
\hline Age at first antipsychotic exposure, years & $32.4[26.6-37.1]$ & $30.8[26.2-35.8]$ \\
\hline Age at schizophrenia onset, years & $32.3[27.1-37.6]$ & $32.5[26.7-37.6]$ \\
\hline Age at DKA onset/index date, years & $37.7[30.7-44.6]$ & $36.8[29.5-44.2]$ \\
\hline Schizophrenia duration, years & $14.1[9.3-15.7]$ & $14.7[12.3-16.6]$ \\
\hline \multicolumn{3}{|l|}{ Schizophrenia ICD-10 subtype } \\
\hline Paranoid (F20.0) & $20(71.4)$ & $83(60.6)$ \\
\hline Other (F20.1-F20.8) & $6(21.4)$ & $31(22.6)$ \\
\hline Unspecified (F20.9) & $2(7.1)$ & $23(16.8)$ \\
\hline \multicolumn{3}{|l|}{ Diabetes aetiology of DKA } \\
\hline Undetermined & $6(21.4)$ & - \\
\hline Post-DKA type 1 diabetes & $8(28.6)$ & - \\
\hline Post-DKA type 2 diabetes & $14(50.0)$ & - \\
\hline \multicolumn{3}{|l|}{ CCI score (within 1 year prior to event) } \\
\hline None $(=0)$ & $23(82.1)$ & $128(93.4)$ \\
\hline Low $(=1)$ & $4(14.3)$ & $7(5.1)$ \\
\hline Moderate/high $(\geq 2)$ & $1(3.6)$ & $2(1.5)$ \\
\hline \multicolumn{3}{|c|}{ Antipsychotic exposure (within 3 months prior to event) } \\
\hline Any antipsychotic & $20(71.4)$ & $67(48.9)$ \\
\hline DDD of antipsychotics & $6.1[1.0-31.1]$ & $5.3[0.3-28.0]$ \\
\hline Antipsychotic polypharmacy & $2(7.1)$ & $28(20.4)$ \\
\hline \multicolumn{3}{|l|}{ FGAs } \\
\hline Low-potency ${ }^{\mathrm{a}}$ & $3(10.7)$ & $16(11.7)$ \\
\hline Mid-potency ${ }^{\mathrm{b}}$ & $2(7.1)$ & $15(10.9)$ \\
\hline High-potency ${ }^{\mathrm{c}}$ & $1(3.6)$ & $5(3.6)$ \\
\hline \multicolumn{3}{|l|}{ SGAs } \\
\hline Amisulpride & $1(3.6)$ & $0(0.0)$ \\
\hline Aripiprazole & $0(0.0)$ & $3(2.2)$ \\
\hline Asenapine & $0(0.0)$ & $0(0.0)$ \\
\hline Clozapine & $4(14.3)$ & $7(5.1)$ \\
\hline Olanzapine & $3(10.7)$ & $31(22.6)$ \\
\hline Paliperidone & $0(0.0)$ & $2(1.5)$ \\
\hline Quetiapine & $4(14.3)$ & $8(5.8)$ \\
\hline Risperidone & $4(14.3)$ & $13(9.5)$ \\
\hline Sertindole & $0(0.0)$ & $1(0.7)$ \\
\hline Ziprasidone & $0(0.0)$ & $1(0.7)$ \\
\hline \multicolumn{3}{|c|}{ Co-medication exposure (within 3 months prior to event) } \\
\hline \multicolumn{3}{|c|}{ Diabetogenic } \\
\hline Thiazides & $0(0.0)$ & $0(0.0)$ \\
\hline Beta blockers & $0(0.0)$ & $1(0.7)$ \\
\hline Statins & $1(3.6)$ & $1(0.7)$ \\
\hline Oral contraceptives & $3(10.7)$ & $6(4.4)$ \\
\hline Glucocorticoids & $0(0.0)$ & $0(0.0)$ \\
\hline Calcineurin inhibitors & $0(0.0)$ & $0(0.0)$ \\
\hline \multicolumn{3}{|l|}{ Psychotropic } \\
\hline Antiepileptics & $1(3.6)$ & $5(3.6)$ \\
\hline Anticholinergics & $1(3.6)$ & $17(12.4)$ \\
\hline Lithium & $0(0.0)$ & $1(0.7)$ \\
\hline Benzodiazepines & $7(25.0)$ & $33(24.1)$ \\
\hline Antidepressants & $5(17.9)$ & $34(24.8)$ \\
\hline
\end{tabular}

Values are presented as $n(\%)$ or medians [p25-p75]

${ }^{a}$ Including chlorpromazine, chlorprothixene, levomepromazine, melperone, pipamperone, sulpiride and thioridazine

${ }^{\mathrm{b}}$ Including loxapine, periciazine, perphenazine, prochlorperazine and zuclopenthixol

${ }^{\mathrm{c}}$ Including flupentixol, fluphenazine, haloperidol, penfluridol and pimozide 
Table 2 Characteristics of incident type 1 diabetes cases and matched controls with schizophrenia

\section{Characteristic}

Type 1 diabetes cases $(n=90)$

Controls $(n=410)$

Demographics

Male sex

$57(63.3)$

$272(66.3)$

Female sex

$33(36.7)$

$138(33.7)$

Age at first antipsychotic exposure, years

39.5 [28.4-49.9]

$40.6[28.1-52.6]$

Age at schizophrenia onset, years

$49.1[33.0-62.6]$

Age at type 1 diabetes onset/index date, years

Schizophrenia duration, years

$11.6[7.4-16.2]$

$37.8[27.5-49.4]$

$39.3[28.1-48.8]$

$45.4[31.4-60.6]$

$14.1[9.0-18.1]$

Schizophrenia ICD-10 subtype

Paranoid (F20.0)

Other (F20.1-20.8)

$54(60.0)$

$18(20.0)$

Unspecified (F20.9)

$18(20.0)$

$275(67.1)$

$63(15.4)$

$72(17.6)$

CCI score (within 1 year prior to event)
None $(=0)$
$72(80.0)$
Low $(=1)$
$10(11.1)$
Moderate/high $(\geq 2)$
8 (8.9)

$340(82.9)$

$48(11.7)$

$22(5.4)$

Antipsychotic exposure (within 3 months prior to event)
Any antipsychotic
DDD of antipsychotics

Antipsychotic polypharmacy

FGAs

Low-potency

Mid-potency ${ }^{b}$

High-potency $^{\mathrm{c}}$

SGAs

Amisulpride

Aripiprazole

Asenapine

Clozapine

Olanzapine

Paliperidone

Quetiapine

Risperidone

Sertindole

Ziprasidone
$63(70.0)$

16.2 [2.9-63.2]

$21(23.3)$

16 (17.8)

$17(18.9)$

$6(6.7)$

1 (1.1)

5 (5.6)

$0(0.0)$

5 (5.6)

$22(24.4)$

$0(0.0)$

$7(7.8)$

$11(12.2)$

1 (1.1)

1 (1.1)
$253(61.7)$

11.0 [2.1-39.4]

$82(20.0)$

$60(14.6)$

$57(13.9)$

20 (4.9)

$4(1.0)$

$18(4.4)$

$0(0.0)$

$29(7.1)$

77 (18.8)

$6(1.5)$

$35(8.5)$

$43(10.5)$

$0(0.0)$

$8(2.0)$
Co-medication exposure (within 3 months prior to event)

\section{Diabetogenic}

$\begin{array}{lll}\text { Thiazides } & 3(3.3) & 12(2.9) \\ \text { Beta blockers } & 6(6.7) & 13(3.2) \\ \text { Statins } & 2(2.2) & 11(2.7) \\ \text { Oral contraceptives } & 2(2.2) & 4(1.0) \\ \text { Glucocorticoids } & 9(10.0) & 4(1.0) \\ \text { Calcineurin inhibitors } & 0(0.0) & 0(0.0) \\ \text { chotropic } & & \\ \text { Antiepileptics } & 7(7.8) & 21(5.1) \\ \text { Anticholinergics } & 19(21.1) & 61(14.9) \\ \text { Lithium } & 3(3.3) & 7(1.7) \\ \text { Benzodiazepines } & 28(31.1) & 124(30.2) \\ \text { Antidepressants } & 27(30.0) & 83(20.2)\end{array}$

Values are presented as $n(\%)$ or medians [p25-p75]

${ }^{\mathrm{a}}$ Including chlorpromazine, chlorprothixene, levomepromazine, melperone, pipamperone, sulpiride and thioridazine

${ }^{\mathrm{b}}$ Including loxapine, periciazine, perphenazine, prochlorperazine and zuclopenthixol

${ }^{\mathrm{c}}$ Including flupentixol, fluphenazine, haloperidol, penfluridol and pimozide 
Table 3 Characteristics of incident type 2 diabetes cases and matched controls with schizophrenia

\begin{tabular}{|c|c|c|}
\hline Characteristic & $\begin{array}{l}\text { Type } 2 \text { diabetes cases } \\
(n=2140)\end{array}$ & $\begin{array}{l}\text { Controls } \\
(n=9861)\end{array}$ \\
\hline \multicolumn{3}{|l|}{ Demographics } \\
\hline Male sex & $1109(51.8)$ & $5146(52.2)$ \\
\hline Female sex & $1031(48.2)$ & $4715(47.8)$ \\
\hline Age at first antipsychotic exposure, years & $37.2[29.0-47.1]$ & $36.7[28.5-45.2]$ \\
\hline Age at schizophrenia onset, years & $39.6[30.2-49.9]$ & $38.4[29.6-47.7]$ \\
\hline $\begin{array}{l}\text { Age at type } 2 \text { diabetes onset/index date, } \\
\text { years }\end{array}$ & $47.7[37.8-57.3]$ & $46.6[37.1-55.4]$ \\
\hline Schizophrenia duration, years & $13.1[8.6-16.9]$ & $13.9[9.3-17.3]$ \\
\hline \multicolumn{3}{|l|}{ Schizophrenia ICD-10 subtype } \\
\hline Paranoid (F20.0) & $1440(67.3)$ & $6536(66.3)$ \\
\hline Other (F20.1-F20.8) & $383(17.9)$ & $1787(18.1)$ \\
\hline Unspecified (F20.9) & $317(14.8)$ & $1538(15.6)$ \\
\hline \multicolumn{3}{|l|}{ CCI score (within 1 year prior to event) } \\
\hline None $(=0)$ & $1694(79.2)$ & $8266(83.8)$ \\
\hline Low $(=1)$ & $304(14.2)$ & $1098(11.1)$ \\
\hline Moderate/high $(\geq 2)$ & $142(6.6)$ & $497(5.0)$ \\
\hline \multicolumn{3}{|c|}{ Antipsychotic exposure (within 3 months prior to event) } \\
\hline Any antipsychotic & $1556(72.7)$ & $6107(61.9)$ \\
\hline DDD of antipsychotics & $22.1[5.9-66.4]$ & $14.3[2.5-53.1]$ \\
\hline Antipsychotic polypharmacy & $670(31.3)$ & $2139(21.7)$ \\
\hline \multicolumn{3}{|l|}{ FGAs } \\
\hline Low-potency ${ }^{\mathrm{a}}$ & $407(19.0)$ & $1363(13.8)$ \\
\hline Mid-potency ${ }^{\mathrm{b}}$ & $305(14.3)$ & $1375(13.9)$ \\
\hline High-potency $^{\mathrm{c}}$ & $81(3.8)$ & $392(4.0)$ \\
\hline \multicolumn{3}{|l|}{ SGAs } \\
\hline Amisulpride & $24(1.1)$ & $78(0.8)$ \\
\hline Aripiprazole & $186(8.7)$ & $714(7.2)$ \\
\hline Asenapine & $1(0.0)$ & $5(0.1)$ \\
\hline Clozapine & $215(10.0)$ & $683(6.9)$ \\
\hline Olanzapine & $383(17.9)$ & $1621(16.4)$ \\
\hline Paliperidone & $40(1.9)$ & $95(1.0)$ \\
\hline Quetiapine & $316(14.8)$ & $913(9.3)$ \\
\hline Risperidone & $307(14.3)$ & $1149(11.7)$ \\
\hline Sertindole & $25(1.2)$ & $63(0.6)$ \\
\hline Ziprasidone & $81(3.8)$ & $271(2.7)$ \\
\hline \multicolumn{3}{|c|}{ Co-medication exposure (within 3 months prior to event) } \\
\hline \multicolumn{3}{|c|}{ Diabetogenic } \\
\hline Thiazides & $169(7.9)$ & $269(2.7)$ \\
\hline Beta blockers & $179(8.4)$ & $380(3.9)$ \\
\hline Statins & $288(13.5)$ & $442(4.5)$ \\
\hline Oral contraceptives & $68(3.2)$ & $384(3.9)$ \\
\hline Glucocorticoids & $42(2.0)$ & $87(0.9)$ \\
\hline Calcineurin inhibitors & $0(0.0)$ & $0(0.0)$ \\
\hline \multicolumn{3}{|l|}{ Psychotropic } \\
\hline Antiepileptics & $209(9.8)$ & $753(7.6)$ \\
\hline Anticholinergics & $347(16.2)$ & $1506(15.3)$ \\
\hline Lithium & $22(1.0)$ & $145(1.5)$ \\
\hline Benzodiazepines & $715(33.4)$ & $2740(27.8)$ \\
\hline Antidepressants & $722(33.7)$ & $2486(25.2)$ \\
\hline
\end{tabular}

Values are presented as $n(\%)$ or medians [p25-p75]

${ }^{a}$ Including chlorpromazine, chlorprothixene, levomepromazine, melperone, pipamperone, sulpiride and thioridazine

${ }^{\mathrm{b}}$ Including loxapine, periciazine, perphenazine, prochlorperazine and zuclopenthixol

${ }^{\mathrm{c}}$ Including flupentixol, fluphenazine, haloperidol, penfluridol and pimozide 


\section{a}

Crude model

Model 1

Model 2

Model 3

b

Crude model

Model 1

Model 2

Model 3

C

Crude model

Model 1

Model 2

Model 3
OR $(95 \% \mathrm{Cl})$

$2.60(1.06,6.38)$

$2.58(1.04,6.40)$

$2.65(1.06,6.59)$

$4.06(1.48,11.11)$

OR $(95 \% \mathrm{Cl})$

$1.38(0.84,2.29)$

$1.38(0.83,2.28)$

$1.32(0.79,2.20)$

$1.16(0.66,2.03)$

OR $(95 \% \mathrm{Cl})$

$1.64(1.48,1.83)$

$1.64(1.47,1.82)$

$1.49(1.34,1.67)$

$1.51(1.34,1.69)$

\section{$p$ value \\ 0.036 \\ 0.041 \\ 0.037 \\ 0.006}

$p$ value
0.206
0.211
0.290
0.613
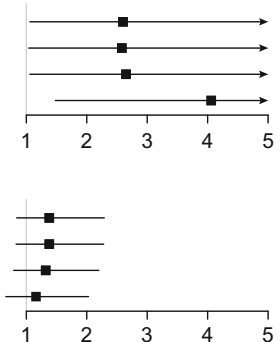

$p$ value

$<0.001$

$<0.001$

$<0.001$

$<0.001$

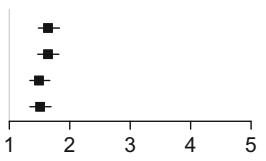

Fig. 2 Conditional logistic regression of risks of DKA and diabetes. Data depict results of conditional logistic regression of associations between antipsychotic exposure (within 3 months prior to event) and DKA (a), type 1 diabetes (b) or type 2 diabetes (c). The crude model is only based on matching by age, sex and year of schizophrenia onset; model 1 is adjusted for low $(=1)$ and moderate/high $(\geq 2)$ CCI score (within 1 year prior to event); model 2 is adjusted for exposure to diabetogenic comedications (within 3 months prior to event); and model 3 is adjusted for exposure to psychotropic co-medications (within 3 months prior to event). In models 2 and 3, co-medication exposure was used as a categorical variable only including integer numbers (e.g. two co-medications yielded twice the listed OR estimate)

cumulative DDD of 1218.8 (p25-p75: 300.0-4556.3). Among the DKA cohort $(n=28)$, of the 14 individuals with post-DKA type 2 diabetes, three $(10.7 \%)$ initially received insulin monotherapy and later switched to monotherapy with oral glucoselowering agents, three $(10.7 \%)$ received monotherapy with oral glucose-lowering agents and eight $(28.6 \%)$ received polytherapy. Median cumulative DDDs of insulin and oral glucose-lowering agents were 1462.5 (p25-p75: 937.54612.5) and 1201.0 (p25-p75: 545.0-1674.0), respectively.

All of the 90 individuals with type 1 diabetes received insulin monotherapy, with a median cumulative DDD of 637.5 (p25-p75: 150.0-1875.0). Of the 2140 individuals with type 2 diabetes, $20(0.9 \%)$ initially received insulin monotherapy and later switched to monotherapy with oral glucose-lowering agents, 1709 (79.9\%) received monotherapy with oral glucoselowering agents and $411(19.2 \%)$ received polytherapy. Median cumulative DDDs of insulin and oral glucose-lowering agents were 825.0 (p25-p75: 187.5-2475.0) and 829.0 (p25-p75: 269.8-2258.0), respectively.

\section{Discussion}

This study investigated associations between antipsychotic exposure (within 3 months prior to event) and DKA, type 1 diabetes and type 2 diabetes, and reported related, clinically relevant outcomes among a previously diabetes-naive schizophrenia population identified through Danish nationwide healthcare registers. Despite the rarity of DKA, with only 28 cases identified between 1995 and 2014, antipsychotic exposure was associated with a nearly threefold increased risk of DKA relative to non-exposure. Of all DKA cases, we were unable to determine diabetes aetiology in six cases, as four were fatal within 8 days and, in two cases, no prescriptions were redeemed for insulin and oral glucose-lowering agents following resolution of DKA. Potentially, the latter may reflect low treatment acceptance in a susceptible population, which is also frequent following other acute medical conditions such as myocardial infarction, where sufficient treatment of post-myocardial infarction tends not to be received [22]. However, more than half of the surviving cases exposed to antipsychotics redeemed prescriptions of insulin and oral glucose-lowering agents following resolution of DKA, indicating a diabetic condition. Predominantly, diabetes aetiology of DKA was deemed to be type 2 diabetes; correspondingly, we found that antipsychotic exposure was associated with a nearly twofold increased risk of type 2 diabetes, whereas risk of type 1 diabetes tended to be slightly increased relative to non-exposure.

Development of antipsychotic-associated DKA is an emerging issue that should advise not only psychiatrists but also general practitioners, internal medicine and other physicians who may manage a DKA emergency to distinguish between the acute effects of antipsychotics on glucose metabolism from those on weight gain. Not only may diabetes develop early during the course of treatment, but DKA may also be the initial presentation. In cases of new-onset diabetes presenting as DKA, the admitting physician often needs to consider several precipitating factors. These may include antipsychotic exposure requiring careful reassessment of treatment in consultation with a psychiatrist. Notably, indications of antipsychotic treatment have recently been widened to the non-schizophrenia population [18], and, due to frequent off-label use, the number of exposed and attributable DKA cases is consequently increasing.

The life-threatening potential of DKA is also more pronounced with antipsychotic exposure relative to the general population, where mortality is less than $1 \%$ [5]. Considering its silent autopsy nature, fatal DKA is often neglected as a potential cause of sudden, unexpected death [23], and the risk of mortality may even be higher than reported here. Sudden, unexpected death, which may be associated with antipsychotic exposure, is, however, often considered multifactorial and can also be caused by cardiac adverse effects of antipsychotics [24].

In clinical practice, early identification of diabetic symptoms following initiation of antipsychotic treatment or during up-titration is crucial to ensure immediate and appropriate management to achieve acceptable glycaemic control in the long term [25]. Consequently, all those who are exposed to antipsychotics should be screened for relevant risk factors for diabetes and potentially DKA, including obesity, marked weight gain, prior personal or family history of diabetes, and ethnicity such as African-American, and undergo routine monitoring of weight and metabolic variables. In addition, if 
Table 4 Differences between cases of post-DKA type 1 diabetes vs type 1 diabetes and cases of post-DKA type 2 diabetes vs type 2 diabetes with schizophrenia

\begin{tabular}{|c|c|c|c|c|c|c|}
\hline Characteristic & $\begin{array}{l}\text { Post-DKA type } 1 \\
\text { diabetes cases } \\
(n=8 / 28)\end{array}$ & $\begin{array}{l}\text { Type } 1 \text { diabetes } \\
\text { cases }(n=90)\end{array}$ & $p$ value & $\begin{array}{l}\text { Post-DKA type } 2 \\
\text { diabetes cases } \\
(n=14 / 28)\end{array}$ & $\begin{array}{l}\text { Type } 2 \text { diabetes } \\
\text { cases }(n=2140)\end{array}$ & $p$ value \\
\hline \multicolumn{7}{|l|}{ Demographics } \\
\hline Male sex & $7(87.5)$ & $57(63.3)$ & 0.323 & $9(64.3)$ & $1109(51.8)$ & 0.508 \\
\hline Female sex & $1(12.5)$ & $33(36.7)$ & 0.256 & $5(35.7)$ & $1031(48.2)$ & 0.508 \\
\hline Age at first antipsychotic exposure, years & $30.8[22.3-30.9]$ & $39.5[28.4-49.9]$ & 0.128 & $32.8[28.0-36.5]$ & $37.2[29.0-47.1]$ & 0.090 \\
\hline Age at schizophrenia onset, years & $30.6[23.6-39.1]$ & $40.6[28.1-52.6]$ & 0.058 & $32.3[27.9-34.9]$ & $39.6[30.2-49.9]$ & 0.012 \\
\hline Age at diabetes onset, years & $35.4[29.2-44.9]$ & $49.1[33.0-62.6]$ & 0.062 & $36.2[31.7-40.9]$ & $47.7[37.8-57.3]$ & 0.002 \\
\hline Schizophrenia duration, years & $14.6[13.1-16.6]$ & $11.6[7.4-16.2]$ & 0.299 & $14.1[12.7-15.2]$ & $13.1[8.6-16.9]$ & 0.428 \\
\hline \multicolumn{7}{|l|}{ Schizophrenia ICD-10 subtype } \\
\hline Paranoid (F20.0) & $5(62.5)$ & $54(60.0)$ & 1.000 & $11(78.6)$ & $1440(67.3)$ & 0.541 \\
\hline Other (F20.1-F20.8) & $3(37.5)$ & $18(20.0)$ & 0.363 & $2(14.3)$ & $383(17.9)$ & 1.000 \\
\hline Unspecified (F20.9) & $0(0.0)$ & $18(20.0)$ & 0.344 & $1(7.1)$ & $317(14.8)$ & 0.707 \\
\hline \multicolumn{7}{|l|}{ CCI score (within 1 year prior to event) } \\
\hline None $(=0)$ & $7(87.5)$ & $72(80.0)$ & 0.962 & $13(92.9)$ & $1694(79.2)$ & 0.353 \\
\hline Low $(=1)$ & $0(0.0)$ & $10(11.1)$ & 1.000 & $1(7.1)$ & $304(14.2)$ & 0.707 \\
\hline Moderate/high $(\geq 2)$ & $1(12.5)$ & $8(8.9)$ & 0.551 & $0(0.0)$ & $142(6.6)$ & 1.000 \\
\hline \multicolumn{7}{|c|}{ Antipsychotic exposure (within 3 months prior to event) } \\
\hline Any antipsychotic & $5(62.5)$ & $63(70.0)$ & 0.967 & $10(71.4)$ & $1556(72.7)$ & 1.000 \\
\hline DDD of antipsychotics & $3.2[0.0-11.3]$ & $16.2[2.9-63.2]$ & 0.055 & $5.0[1.0-9.7]$ & $22.1[5.9-66.4]$ & 0.012 \\
\hline Antipsychotic polypharmacy & $0(0.0)$ & $21(23.3)$ & 0.196 & $1(7.1)$ & $670(31.3)$ & 0.078 \\
\hline \multicolumn{7}{|l|}{ FGAs } \\
\hline Low-potency ${ }^{\mathrm{a}}$ & $0(0.0)$ & $16(17.8)$ & 0.346 & $2(14.3)$ & $407(19.0)$ & 1.000 \\
\hline Mid-potency ${ }^{\mathrm{b}}$ & $1(12.5)$ & $17(18.9)$ & 1.000 & $1(7.1)$ & $305(14.3)$ & 0.707 \\
\hline High-potency $^{c}$ & $0(0.0)$ & $6(6.7)$ & 1.000 & $0(0.0)$ & $81(3.8)$ & 1.000 \\
\hline \multicolumn{7}{|l|}{ SGAs } \\
\hline Amisulpride & $0(0.0)$ & $1(1.1)$ & 1.000 & $1(7.1)$ & $24(1.1)$ & 0.151 \\
\hline Aripiprazole & $0(0.0)$ & $5(5.6)$ & 1.000 & $0(0.0)$ & $186(8.7)$ & 0.625 \\
\hline Asenapine & $0(0.0)$ & $0(0.0)$ & - & $0(0.0)$ & $1(0.0)$ & 1.000 \\
\hline Clozapine & $2(25.0)$ & $5(5.6)$ & 0.100 & $2(14.3)$ & $215(10.0)$ & 0.645 \\
\hline Olanzapine & $0(0.0)$ & $22(24.4)$ & 0.193 & $1(7.1)$ & 383 (17.9) & 0.487 \\
\hline Paliperidone & $0(0.0)$ & $0(0.0)$ & - & $0(0.0)$ & $40(1.9)$ & 1.000 \\
\hline Quetiapine & $0(0.0)$ & $7(7.8)$ & 1.000 & $2(14.3)$ & $316(14.8)$ & 1.000 \\
\hline Risperidone & $2(25.0)$ & $11(12.2)$ & 0.286 & $2(14.3)$ & 307 (14.3) & 1.000 \\
\hline Sertindole & $0(0.0)$ & $1(1.1)$ & 1.000 & $0(0.0)$ & $25(1.2)$ & 1.000 \\
\hline Ziprasidone & $0(0.0)$ & $1(1.1)$ & 1.000 & $0(0.0)$ & $81(3.8)$ & 1.000 \\
\hline \multicolumn{7}{|c|}{ Co-medication exposure (within 3 months prior to event) } \\
\hline \multicolumn{7}{|l|}{ Diabetogenic } \\
\hline Thiazides & $0(0.0)$ & $3(3.3)$ & 1.000 & $0(0.0)$ & $169(7.9)$ & 0.619 \\
\hline Beta blockers & $0(0.0)$ & $6(6.7)$ & 1.000 & $0(0.0)$ & $179(8.4)$ & 0.622 \\
\hline Statins & $1(12.5)$ & $2(2.2)$ & 0.228 & $0(0.0)$ & $288(13.5)$ & 0.239 \\
\hline Oral contraceptives & $0(0.0)$ & $2(2.2)$ & 1.000 & $3(21.4)$ & $68(3.2)$ & 0.010 \\
\hline Glucocorticoids & $0(0.0)$ & $9(10.0)$ & 1.000 & $0(0.0)$ & $42(2.0)$ & 1.000 \\
\hline Calcineurin inhibitors & $0(0.0)$ & $0(0.0)$ & - & $0(0.0)$ & $0(0.0)$ & - \\
\hline \multicolumn{7}{|l|}{ Psychotropic } \\
\hline Antiepileptics & $0(0.0)$ & $7(7.8)$ & 1.000 & $0(0.0)$ & $209(9.8)$ & 0.386 \\
\hline Anticholinergics & $1(12.5)$ & $19(21.1)$ & 1.000 & $0(0.0)$ & $347(16.2)$ & 0.145 \\
\hline Lithium & $0(0.0)$ & $3(3.3)$ & 1.000 & $0(0.0)$ & $22(1.0)$ & 1.000 \\
\hline
\end{tabular}


Table 4 (continued)

\begin{tabular}{|c|c|c|c|c|c|c|}
\hline Characteristic & $\begin{array}{l}\text { Post-DKA type } 1 \\
\text { diabetes cases } \\
(n=8 / 28)\end{array}$ & $\begin{array}{l}\text { Type } 1 \text { diabetes } \\
\text { cases }(n=90)\end{array}$ & $p$ value & $\begin{array}{l}\text { Post-DKA type } 2 \\
\text { diabetes cases } \\
(n=14 / 28)\end{array}$ & $\begin{array}{l}\text { Type } 2 \text { diabetes } \\
\text { cases }(n=2140)\end{array}$ & $p$ value \\
\hline Benzodiazepines & $1(12.5)$ & $28(31.1)$ & 0.430 & $2(14.3)$ & 715 (33.4) & 0.162 \\
\hline Antidepressants & $1(12.5)$ & $27(30.0)$ & 0.433 & $4(28.6)$ & $722(33.7)$ & 0.784 \\
\hline
\end{tabular}

Values are presented as $n(\%)$ or medians [p25-p75]

$p$ values are based on the non-parametric Mann-Whitney $U$ test for continuous variables and on Fisher's exact or the $\chi^{2}$ test for categorical variables for fewer than five cases and for five or more cases, respectively

Of the remaining six of 28 DKA cases, the aetiology could not be determined, as four were fatal within 8 days and two were not redeeming prescriptions for insulin and oral glucose-lowering agents

${ }^{a}$ Including chlorpromazine, chlorprothixene, levomepromazine, melperone, pipamperone, sulpiride and thioridazine

${ }^{\mathrm{b}}$ Including loxapine, periciazine, perphenazine, prochlorperazine and zuclopenthixol

${ }^{\mathrm{c}}$ Including flupentixol, fluphenazine, haloperidol, penfluridol and pimozide

clinically feasible, low-risk metabolic antipsychotics should preferentially be prescribed [6].

Most antipsychotics were at risk of causing DKA but due to the limited number of cases ORs for individual antipsychotics could not be estimated. However, cases exposed to high-risk metabolic antipsychotics, including clozapine and olanzapine, relative to those of low risk, including aripiprazole, ziprasidone and high-potency FGAs, had an increased incidence of DKA, thus corroborating the findings of previous studies [7, 26, 27] and reviews [28-31]. However, confounding by indication could underestimate the risk of DKA associated with highrisk metabolic antipsychotics, as physicians may be less likely to prescribe them to someone with known risk factors (see above). Conversely, the effect of clozapine may be overestimated, as it is indicated for refractory schizophrenia and reserved for the most severely ill [32]. This could affect the risk of DKA through unhealthy lifestyle habits causing weight gain during the course of treatment and through neglect of early diabetic symptoms by not seeking medical attention. Among those exposed to clozapine, a detection bias could further exist, as frequent blood draws are a part of the mandatory haematological monitoring that would more readily detect diabetes. This is quite important, as increased $\mathrm{HbA}_{1 \mathrm{c}}$ levels are frequently reported at admission for DKA in the schizophrenia population exposed to antipsychotics [7].

While physical comorbidity, reflected by increased CCI score, and exposure to diabetogenic co-medications did not affect the risk of DKA, exposure to psychotropic co-medications increased the risk by fourfold. Using various combinations of psychotropic medications with antipsychotics is often necessary to control severe symptoms of schizophrenia and potentially reduce psychiatric admissions and antipsychotic use [33]. However, this may also cause unwarranted polypharmacy, drug-drug interactions and, consequently, overlapping associated adverse effects. Thus, physicians should keep in mind that DKA may be associated with other psychotropic medications, including lithium [29] and antidepressants [34], with the latter being associated with increased risk of mortality [35]. In addition, our study underscores that development of DKA is not only an issue with SGAs due to potential metabolic liabilities, which most physicians may worry about, but also with FGAs. In addition, only a few cases have been previously reported regarding DKA associated with FGAs [29]. That the low-risk metabolic antipsychotic aripiprazole may cause DKA further fuels the argument of more acute effects of antipsychotics on beta cell function $[28,29]$. Although aripiprazole is less likely to cause weight gain and glucose dysregulation [20], confounding by indication cannot be ruled out, as those at high risk tend to have aripiprazole prescribed for safety reasons [36]. Conversely, we did not identify any DKA cases associated with aripiprazole. However, weight gain during the course of treatment cannot be ignored as a significant risk factor. A previous study found that olanzapine relative to risperidone increased the risk of DKA by 1.7 -fold following over 30 days of exposure and by 3.5-fold following over 180 days [26].

Of further concern, the fact that some cases developed DKA while not exposed to antipsychotics also warrants discussion. Schizophrenia per se is associated with glucose dysregulation $[37,38]$, and psychiatric decompensation may promote release of glucose counterregulatory hormones [28, 39], which, consequently, increases the risk of DKA in the presence of reduced beta cell function (and antipsychotic exposure). Here, unhealthy lifestyle habits may also lead to the onset of diabetes and potentially DKA [2]. Level of education and integration in society may further play a role in the diagnostic delay and high rate of DKA readmissions, as DKA is over-represented among minority groups, where social deprivation is frequent $[35,40]$.

Diabetes aetiology of DKA was deemed to be type 2 diabetes, which is the most widely recognised and established aetiology in the schizophrenia population [29-31], in contrast to type 1 diabetes, which is more speculative [28]. Correspondingly, the risk of type 2 diabetes associated with antipsychotic exposure was increased, thus corroborating the findings of a previous study 

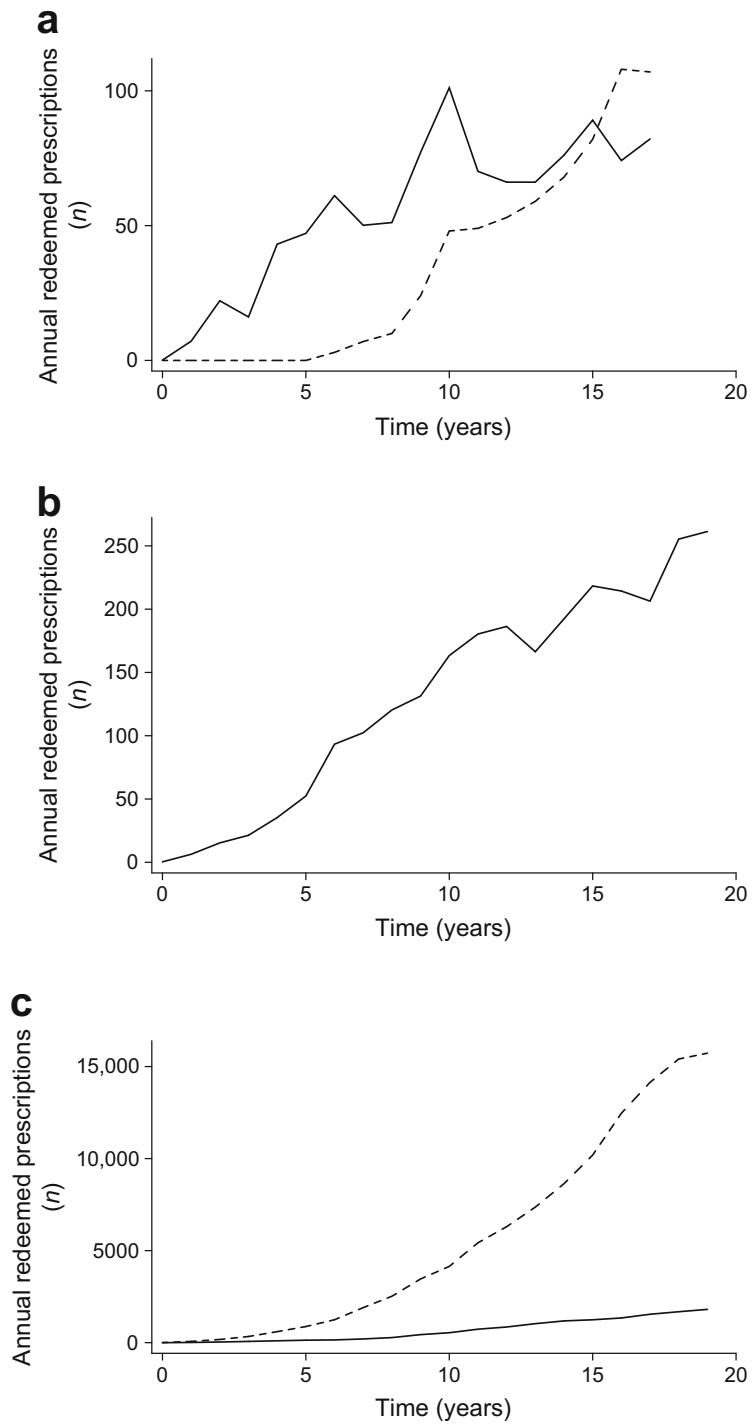

Fig. 3 Temporal trends of use of insulin and oral glucose-lowering agents. The figure depicts annual redeemed prescriptions of insulin (solid line) and oral glucose-lowering agents (dashed line) following incident onsets of DKA (a), type 1 diabetes (b) and type 2 diabetes (c) until 31 December 2014

[20]. While the risk of type 1 diabetes associated with antipsychotic exposure tended to be slightly increased, although insignificant, the risk of type 1 diabetes in the schizophrenia population is reduced [8-11].

Some type 2 diabetes cases and post-DKA type 2 diabetes cases initially received insulin and later switched to oral glucose-lowering agents, potentially reflecting the physician's judgement that the individual had either type 1 diabetes or ketosis-prone type 2 diabetes, where both insulin secretion and action are transitorily impaired [5]. With high-dose insulin treatment, improvement in beta cell function and insulin sensitivity may be achieved, and insulin can slowly be discontinued within a few months, achieving acceptable glycaemic control with lifestyle modification and/or oral glucose-lowering agents. Another diagnostic pitfall also includes latent autoimmune diabetes in adults (LADA), which is defined by onset around 30 years, presence of type 1 diabetes autoantibodies, and a period without requirement of insulin treatment of at least 6 months (i.e. honeymoon period) [41].

A major limitation of this study is the observational design, meaning that our findings represent associations and not necessarily causal inferences. In addition, unmeasured and unknown potential confounders, including family history of diabetes, ethnicity, unhealthy lifestyle, BMI, severity of schizophrenia, medication adherence and changes in prescribing patterns, might have biased our findings. Unfortunately, we did not have access to such information. Although the validity of Danish nationwide healthcare registers has proven to be high [42], we were not able to validate the diagnosis of DKA using blood samples to distinguish from other similar metabolic emergencies, including hyperosmolar hyperglycaemic state or lactic acidosis. In addition, we used a pragmatic definition to distinguish between type 1 diabetes and type 2 diabetes, relying on redeemed prescriptions for insulin and oral glucose-lowering agents, which unfortunately may not apply to all cases. Consequently, we were not able to identify primarily those with type 2 diabetes who were only treated with lifestyle modification rather than with insulin and oral glucose-lowering agents.

Although we only investigated the influence of antipsychotic exposure within 3 months prior to event, cumulative exposure may have contributed to our findings due to carryover effects. In addition, we used a non-exposure population as reference, knowing that this heterogeneous population may affect our findings, as it differs in physical health status and access to healthcare. Potentially, this population could comprise those with a more benign course of schizophrenia where antipsychotic treatment is unwarranted, those who are homeless or imprisoned and the most severely ill where treatment acceptance is particularly low. The control population may also consist of individuals receiving antipsychotic medication free of charge (i.e. the newly diagnosed, those treated by forensic psychiatrists and/or those participating in research studies). However, we used this suboptimal approach, as choosing an individual antipsychotic as reference would cause confounding by indication, making the interpretation even more difficult.

Conclusions DKA is an important adverse effect associated with recent antipsychotic exposure and may have a fatal course among a previously diabetes-naive schizophrenia population. Despite the rarity, psychiatrists, as well as other physicians, should be aware of prevention, early recognition and treatment of antipsychotic-associated DKA. It is crucial to determine as quickly as possible the diabetes aetiology of DKA; diagnostic pitfalls may include ketosis-prone type 2 diabetes and LADA. Antipsychotic exposure may also increase the risk of type 2 diabetes, whereas the association with type 1 diabetes is rather unclear and warrants further investigation. 
Data availability Data sharing is not applicable to this study as data management and analysis were performed on a Statistics Denmark server through remote access, for privacy and safety concerns.

Funding This study received no specific grant from any funding agency in the public, commercial or not-for-profit sectors.

Duality of interest CP has received speaking fees from $\mathrm{H}$. Lundbeck. KK has received speaking fees from Novartis and research grants from the Laerdal Foundation. JN has received speaking fees from BristolMyers Squibb, H. Lundbeck, HemoCue and Sunovion and has received research grants from $\mathrm{H}$. Lundbeck and Pfizer. No other disclosures were reported.

Contribution statement $\mathrm{CP}$ and $\mathrm{KK}$ initiated the study, participated in its design and coordination, and performed the statistical analyses. CR, $\mathrm{NH}, \mathrm{TV}$ and $\mathrm{JN}$ provided subsequent input in developing the study design and contributing to data collection, analysis and interpretation. $\mathrm{CP}$ wrote the initial draft of the manuscript, with revisions provided by all authors. The final manuscript was approved by all authors. $\mathrm{CP}$ is the guarantor of this study and, as such, had full access to all the data in the study and takes responsibility for the integrity of the data and the accuracy of the statistical analysis.

\section{References}

1. Laursen TM, Nordentoft M, Mortensen PB (2014) Excess early mortality in schizophrenia. Annu Rev Clin Psychol 10:425-448

2. Henderson DC, Vincenzi B, Andrea NV, Ulloa M, Copeland PM (2015) Pathophysiological mechanisms of increased cardiometabolic risk in people with schizophrenia and other severe mental illnesses. Lancet Psychiatry 2:452-464

3. Roberts L, Roalfe A, Wilson S, Lester H (2007) Physical health care of patients with schizophrenia in primary care: a comparative study. Fam Pract 24:34-40

4. Marder SR, Essock SM, Miller AL et al (2004) Physical health monitoring of patients with schizophrenia. Am J Psychiatry 161: 1334-1349

5. Nyenwe EA, Kitabchi AE (2016) The evolution of diabetic ketoacidosis: an update of its etiology, pathogenesis and management. Metabolism 65:507-521

6. Cohen D, Correll CU (2009) Second-generation antipsychotic-associated diabetes mellitus and diabetic ketoacidosis: mechanisms, predictors, and screening need. J Clin Psychiatry 70:765-766

7. Henderson DC, Cagliero E, Copeland PM et al (2007) Elevated hemoglobin A1c as a possible indicator of diabetes mellitus and diabetic ketoacidosis in schizophrenia patients receiving atypical antipsychotics. J Clin Psychiatry 68:533-541

8. Hallford P, Clair DS, Halley L, Mustard C, Wei J (2016) A study of type-1 diabetes associated autoantibodies in schizophrenia. Schizophr Res 176:186-190

9. Juvonen H, Reunanen A, Haukka J et al (2007) Incidence of schizophrenia in a nationwide cohort of patients with type 1 diabetes mellitus. Arch Gen Psychiatry 64:894-899

10. Eaton WW, Byrne M, Ewald $\mathrm{H}$ et al (2006) Association of schizophrenia and autoimmune diseases: linkage of Danish national registers. Am J Psychiatry 163:521-528

11. Cohen D, Batstra MR, Gispen-de Wied CC (2005) Immunological characteristics of diabetes in schizophrenia. Diabetologia 48:1941-1942

12. Galler A, Bollow E, Meusers M et al (2015) Comparison of glycemic and metabolic control in youth with type 1 diabetes with and without antipsychotic medication: analysis from the nationwide
German/Austrian Diabetes Survey (DPV). Diabetes Care 38: 1051-1057

13. Pedersen CB (2011) The Danish Civil Registration System. Scand J Public Health 39:22-25

14. Mors O, Perto GP, Mortensen PB (2011) The Danish Psychiatric Central Research Register. Scand J Public Health 39:54-57

15. Lynge E, Sandegaard JL, Rebolj M (2011) The Danish National Patient Register. Scand J Public Health 39:30-33

16. Kildemoes HW, Sørensen HT, Hallas J (2011) The Danish National Prescription Registry. Scand J Public Health 39:38-41

17. Helweg-Larsen K (2011) The Danish Register of Causes of Death. Scand J Public Health 39:26-29

18. Maher AR, Maglione M, Bagley S et al (2011) Efficacy and comparative effectiveness of atypical antipsychotic medications for offlabel uses in adults: a systematic review and meta-analysis. JAMA 306:1359-1369

19. Mor A, Berencsi K, Svensson E et al (2015) Prescribing practices and clinical predictors of glucose-lowering therapy within the first year in people with newly diagnosed type 2 diabetes. Diabet Med 32:1546-1554

20. Nielsen J, Skadhede S, Correll CU (2010) Antipsychotics associated with the development of type 2 diabetes in antipsychotic-naive schizophrenia patients. Neuropsychopharmacology 35:1997-2004

21. Charlson ME, Pompei P, Ales KL, MacKenzie CR (1987) A new method of classifying prognostic comorbidity in longitudinal studies: development and validation. J Chronic Dis 40:373-383

22. Nielsen J, Juel J, Alzuhairi KS et al (2015) Unrecognised myocardial infarction in patients with schizophrenia. Acta Neuropsychiatr 27:106-112

23. Ali Z, Levine B, Ripple M, Fowler DR (2012) Diabetic ketoacidosis: a silent death. Am J Forensic Med Pathol 33:189-193

24. Polcwiartek C, Kragholm K, Schjerning O, Graff C, Nielsen J (2016) Cardiovascular safety of antipsychotics: a clinical overview. Expert Opin Drug Saf 15:679-688

25. Doherty AM (2015) Psychiatric aspects of diabetes mellitus. Br J Psychiatr Adv 21:407-416

26. Ramaswamy K, Kozma CM, Nasrallah H (2007) Risk of diabetic ketoacidosis after exposure to risperidone or olanzapine. Drug Saf 30:589-599

27. Leslie DL, Rosenheck RA (2004) Incidence of newly diagnosed diabetes attributable to atypical antipsychotic medications. Am J Psychiatry 161:1709-1711

28. Polcwiartek C, Vang T, Bruhn $\mathrm{CH}$, Hashemi N, Rosenzweig M, Nielsen J (2016) Diabetic ketoacidosis in patients exposed to antipsychotics: a systematic literature review and analysis of Danish adverse drug event reports. Psychopharmacology 233:3663-3672

29. Guenette MD, Hahn M, Cohn TA, Teo C, Remington GJ (2013) Atypical antipsychotics and diabetic ketoacidosis: a review. Psychopharmacology 226:1-12

30. Cohen D (2004) Atypical antipsychotics and new onset diabetes mellitus. An overview of the literature. Pharmacopsychiatry 37:1-11

31. Jin H, Meyer JM, Jeste DV (2002) Phenomenology of and risk factors for new-onset diabetes mellitus and diabetic ketoacidosis associated with atypical antipsychotics: an analysis of 45 published cases. Ann Clin Psychiatry 14:59-64

32. Nielsen J, Dahm M, Lublin H, Taylor D (2010) Psychiatrists' attitude towards and knowledge of clozapine treatment. J Psychopharmacol 24:965-971

33. Rohde C, Polcwiartek C, Asztalos M, Nielsen J (2017) Effectiveness of prescription-based CNS stimulants on hospitalization in patients with schizophrenia: a nation-wide register study. Schizophr Bull doi:10.1093/schbul/sbx043

34. Barnard K, Peveler RC, Holt RI (2013) Antidepressant medication as a risk factor for type 2 diabetes and impaired glucose regulation: systematic review. Diabetes Care 36:3337-3345 
35. Gibb FW, Teoh WL, Graham J, Lockman KA (2016) Risk of death following admission to a UK hospital with diabetic ketoacidosis. Diabetologia 59:2082-2087

36. Polcwiartek C, Sneider B, Graff C et al (2015) The cardiac safety of aripiprazole treatment in patients at high risk for torsade: a systematic review with a meta-analytic approach. Psychopharmacology 232:3297-3308

37. Rajkumar AP, Horsdal HT, Wimberley T et al (2017) Endogenous and antipsychotic-related risks for diabetes mellitus in young people with schizophrenia: a Danish population-based cohort study. Am J Psychiatry doi:10.1176/appi.ajp.2016.16040442

38. Pillinger T, Beck K, Gobjila C, Donocik JG, Jauhar S, Howes OD (2017) Impaired glucose homeostasis in first-episode schizophrenia: a systematic review and meta-analysis. JAMA Psychiatry 74:261269
39. Shiloah E, Witz S, Abramovitch Y et al (2003) Effect of acute psychotic stress in nondiabetic subjects on beta-cell function and insulin sensitivity. Diabetes Care 26:1462-1467

40. Usher-Smith JA, Thompson MJ, Sharp SJ, Walter FM (2011) Factors associated with the presence of diabetic ketoacidosis at diagnosis of diabetes in children and young adults: a systematic review. BMJ 343:d4092

41. Laugesen E, Østergaard JA, Leslie RDG (2015) Latent autoimmune diabetes of the adult: current knowledge and uncertainty. Diabet Med 32:843-852

42. Uggerby P, Østergaard SD, Roge R, Correll CU, Nielsen J (2013) The validity of the schizophrenia diagnosis in the Danish Psychiatric Central Research Register is good. Dan Med J 60: A4578 